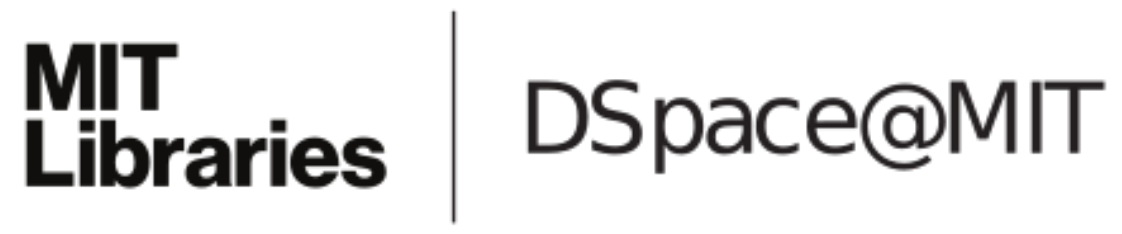

\author{
MIT Open Access Articles
}

Reduced lasing threshold from organic dye microcavities

The MIT Faculty has made this article openly available. Please share how this access benefits you. Your story matters.

Citation: Akselrod, G. M., E. R. Young, K. W. Stone, A. Palatnik, V. Bulovic, and Y. R. Tischler. "Reduced lasing threshold from organic dye microcavities" Phys. Rev. B 90, 035209 (July 2014). (C) 2014 American Physical Society

As Published: http://dx.doi.org/10.1103/PhysRevB.90.035209

Publisher: American Physical Society

Persistent URL: http://hdl.handle.net/1721.1/88664

Version: Final published version: final published article, as it appeared in a journal, conference proceedings, or other formally published context

Terms of Use: Article is made available in accordance with the publisher's policy and may be subject to US copyright law. Please refer to the publisher's site for terms of use. 


\title{
Reduced lasing threshold from organic dye microcavities
}

\author{
G. M. Akselrod, ${ }^{1}$ E. R. Young, ${ }^{1,2}$ K. W. Stone, ${ }^{1}$ A. Palatnik, ${ }^{3}$ V. Bulović, ${ }^{1}$ and Y. R. Tischler ${ }^{3, *}$ \\ ${ }^{1}$ Center for Excitonics, Massachusetts Institute of Technology, Cambridge, Massachusetts 02139-4307, USA \\ ${ }^{2}$ Department of Chemistry, Amherst College, Amherst, Massachusetts 01002-5000, USA \\ ${ }^{3}$ Department of Chemistry, Bar-Ilan University, Ramat Gan, Israel
}

(Received 6 April 2014; revised manuscript received 20 May 2014; published 24 July 2014)

\begin{abstract}
We demonstrate an unexpected tenfold reduction in the lasing threshold of an organic vertical microcavity under subpicosecond optical excitation. In contrast to conventional theory of lasing, we find that the lasing threshold depends on the rate at which excitons are created rather than the total energy delivered within the exciton lifetime. The threshold reduction is discussed in the context of microcavity-enhanced super-radiant coupling between the excitons. The interpretation of super-radiance is supported by the temporal relaxation dynamics of the microcavity emission, which follows the super-radiance time rather than the cavity lifetime. This demonstration suggests that room-temperature super-radiant effects could generally lower the threshold in four-level lasing systems of similar relaxation dynamics.
\end{abstract}

DOI: 10.1103/PhysRevB.90.035209

PACS number(s): 42.55.Mv, 42.55.Px, 71.35.-y

\section{INTRODUCTION}

Lasers based on organic molecules as the gain material offer a number of technological advantages, such as broad color tunability, additive nonepitaxial growth on many substrates, and the ability to be used for direct chemical sensing [1]. Organic lasers employing conjugated polymer and molecular organic films have been demonstrated in a wide range of geometries including vertical microcavities [2], slab waveguides [3], and distributed feedback structures [4]. A key pursuit in the research on microcavity organic lasers is to investigate and enhance light-matter interactions. This could lead to reduced lasing thresholds, possibly reaching optical or electrical excitation threshold power densities that can be practically achieved in compact structures. A number of strategies for reducing the lasing threshold have already been explored. The most studied approach is to modify the laser resonator cavity by increasing the reflectivity of the mirrors [5], which increases the effective photon path length, thus leading to a reduced threshold. Alternatively, theory shows that the lasing threshold could be reduced by strongly coupling organic excitons to the photon field of the cavity, forming quasiparticles known as polaritons. Polaritons at high densities can undergo condensation, resulting in coherent laserlike emission from the cavity in a process that is distinct from conventional stimulated emission [6] and is predicted to occur at excitation densities lower than conventional lasing. Polariton lasing in organic films was first demonstrated in a vertical microcavity filled with anthracene [7], and more recently condensation of polaritons in a polymer-filled microcavity was observed [8]. However, despite the progress in demonstrating coherent emission from these strongly coupled structures, substantial reductions in the lasing threshold have not been achieved.

Here we report on threshold reduction in organic vertical microcavities by tailoring the duration of the optical excitation pulse. The gain material consists of a molecular thin film of the archetypal guest-host blend of 4-dicyanmethylene-2methyl-6-( $p$-dimethylaminostyryl)-4H-pyran:aluminum tris-

*yaakov.tischler@biu.ac.il (8-hydroxyquinoline) (DCM:Alq 3 ). In contrast with the predictions of conventional lasing theory, we find that the lasing threshold depends on the rate of excitation, rather than only the total energy delivered within the exciton lifetime. As a possible mechanism responsible for the threshold reduction, we consider the phenomenon of super-radiance (SR) - the coherent coupling of spontaneous emission from emitters at high density [9]. In conventional lasing, threshold occurs when the rate of stimulated emission into the cavity mode is equal to the rate of energy flow out of the cavity, given by [10]

$$
n_{\mathrm{th}} \sigma_{\mathrm{SE}} c=\frac{1}{\tau_{c}},
$$

where $n_{\text {th }}$ is the density of excitons at threshold, $\sigma_{\mathrm{SE}}$ is the stimulated emission cross section, $c$ is the speed of light, and $\tau_{c}$ is the photon lifetime in the cavity. In this expression, the time scale on which the exciton density is created relative to the dephasing time is not considered because the conventional lasing action does not rely on coherence between the excited states of matter, which in our study are excitons in a molecular organic solid-state gain layer [Fig. 1(a)]. However, if the excitons are created at sufficient density and in a time $\tau_{\text {pump }}$, which is much shorter than the spontaneous emission lifetime of the exciton $\tau_{\mathrm{sp}}$, coherent interactions between excitons can result in super-radiant emission [9,11-13]. SR is characterized by a fast, intense, and directional emission pulse with a characteristic time scale $\tau_{R} \ll \tau_{\mathrm{sp}}$. Furthermore, the presence of an optical cavity can increase the SR rate by a factor proportional to the finesse of the cavity by creating a longer effective sample length $[11,14]$.

\section{EXPERIMENTAL METHODS}

The microcavities measured in this study are composed of an organic thin-film gain layer sandwiched between a distributed Bragg reflector (DBR) and an Ag or DBR mirror as shown in Fig. 1(a). For the low- $Q$ cavity, the DBR mirror is formed from 6.5 pairs of sputter-coated $\mathrm{TiO}_{2}$ and $\mathrm{SiO}_{2}$ thin films that serve as the high and low refractive index layers $\left(\eta_{\mathrm{TiO}_{2}}=2.41\right.$ and $\left.\eta_{\mathrm{SiO}_{2}}=1.46\right)$ of thicknesses 62 and $102 \mathrm{~nm}$, respectively. The organic gain layer is a thin film 
(a)

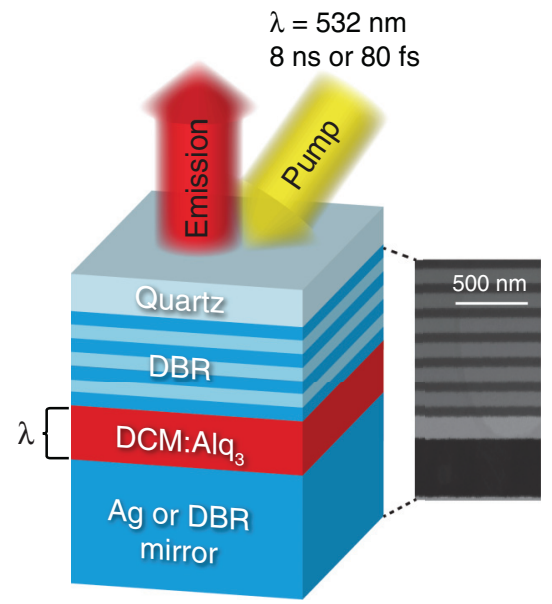

(b)

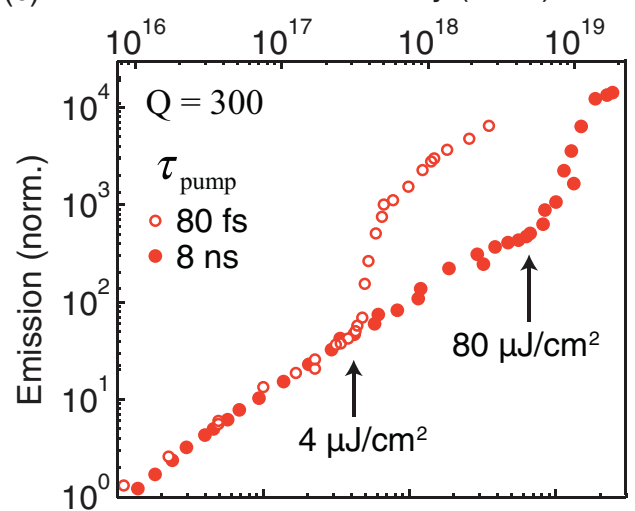

(c)

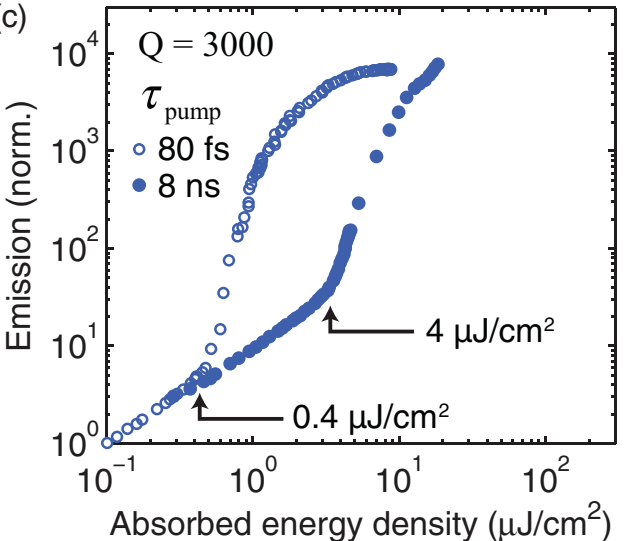

FIG. 1. (Color online) (a) Architecture and cross-sectional scanning electron microscope image of an organic microcavity with DCM gain material situated between a DBR and an Ag mirror (low- $Q$ cavity) or a second DBR mirror (high- $Q$ cavity). (b) and (c) Normalized emission intensity as a function of excitation density for the (b) $Q=300$ and (c) $Q=3000$ microcavities, showing different thresholds under pulsed excitation with durations of $80 \mathrm{fs}$ and $8 \mathrm{~ns}$.

of laser dye DCM doped at $2.8 \%$ by weight into $\mathrm{Alq}_{3}$ host material where the mixed DCM:Alq 3 film is deposited by thermal coevaporation onto the DBR mirror. The $\mathrm{Alq}_{3}$ is a passive host in our system because the DCM is excited directly with $\lambda=532-\mathrm{nm}$ wavelength light, that is not absorbed by $\mathrm{Alq}_{3}$. DCM is a four-level laser dye with a stimulated emission cross section of $\sigma_{\mathrm{SE}}=1.1 \times 10^{-16} \mathrm{~cm}^{2}$ [10], a spontaneous emission lifetime of $\tau_{\mathrm{sp}}=3 \mathrm{~ns}$, and a broad $\left(\Delta \lambda_{\mathrm{DCM}}=80-\mathrm{nm}\right)$ luminescence emission spectrum centered at $\lambda_{\mathrm{DCM}}=620 \mathrm{~nm}$. The gain layer thickness is varied across the sample to allow for probing at the desired cavity tuning of $\lambda=600 \pm 5 \mathrm{~nm}$. The 500-nm-thick Ag mirror is deposited by thermal evaporation onto the gain layer, resulting in a cavity with a quality factor of $Q=300$. The cavity photon lifetime is $95 \mathrm{fs}$, derived from the relation $\tau_{c}=Q \lambda / 2 \pi c$, where $Q$ is obtained from emission spectra $(\Delta \lambda=2 \mathrm{~nm})$ and is confirmed by transfer-matrix modeling [15].

Due to the difficulty of sputtering dielectric mirrors on organic thin films, the high- $Q$ cavity $(Q=3000$ ) is fabricated by a stamping technique. DBRs with 14.5 pairs of $\mathrm{SiO}_{2}$ and $\mathrm{Ta}_{2} \mathrm{O}_{5}$ are commercially grown on flat substrates and on substrates with a $10-\mathrm{m}$ radius of curvature. DCM: $\mathrm{Alq}_{3}$ is deposited on the flat substrate as described above, and the curved DBR mirror is aligned, pressed, and sealed onto the flat substrate, forming the $\lambda$-thickness microcavity. Photon lifetime for the high- $Q$ cavity is $1300 \mathrm{fs}(\Delta \lambda=0.15 \mathrm{~nm})$.

The microcavities are optically excited through the substrate with a $T_{M}$ polarized pump beam, incident at $\theta=60^{\circ}$ from normal, with the excitation focused to a spot size of $300 \mu \mathrm{m}$ in diameter. To vary the duration of the excitation pulses, three pump lasers are used: frequency-doubled $Q$ switched Nd:YAG lasers emitting 0.9- or 8-ns pulses at $\lambda_{\mathrm{ex}}=$ $532 \mathrm{~nm}$ and a mode-locked regeneratively amplified laser system that pumps an optical parametric amplifier to deliver $\sim 80$-fs duration pulses at $\lambda=532 \mathrm{~nm}$. All laser repetition rates are $\sim 1 \mathrm{kHz}$. The duration of the 80 -fs pulses is varied using a grating-based pulse stretcher with spectral windowing [16]. Cavity emission spectra are collected via an optical fiber coupled into a spectrograph and imaged onto a CCD camera. The excitation wavelength in all cases is tuned in order to pump DCM molecules and to avoid excitation of $\mathrm{Alq}_{3}$ with on average $25 \%$ of the incident light absorbed in the cavity. Due to fast subpicosecond Franck-Condon relaxation of excited DCM molecules to the metastable excited state [17], the instantaneous hot exciton density can be considered equal to the emissive state exciton density.

For all measurements other than the time-resolved data, the excitation spot size is determined by scanning a knife edge across the focused excitation beam at the position of the sample. The obtained spot diameter is $300 \mu \mathrm{m}$ at the $1 / e$ intensity points. To obtain the incident energy density per pulse on the sample, the average power of the incident beam is measured using a calibrated Si photodetector (Ophir-1Z02413 with power meter Ophir-1Z01803). The measurement range of the power meter is chosen such that the detector is not saturated due to the pulsed excitation. To verify the accuracy of the power measurements, we measure the power with a calibrated thermopile detector (Newport 818P-010-12 with power meter 1918-C), which in contrast to a photodiode is not susceptible to pulsed excitation saturation. The observed agreement between the two types of measurements allowed us to use the Ophir Si photodetector for the low excitation powers needed for cavity experiments.

The fraction of incident light that is absorbed in the gain medium is obtained by measuring the power of the excitation light reflected by the sample compared with the power incident 
on the sample. The back mirror of the cavity is a 500-nm-thick Ag layer, and the bottom DBR mirror has low optical losses. Hence, any light not reflected by the sample is absorbed by the gain layer. At an angle of incidence of $60^{\circ}$ relative to normal, the typical absorption of the cavity at the excitation wavelength of $532 \mathrm{~nm}$ is $25 \%$. By combining the measurements of spot size, incident power, and reflected power, we obtain the absorbed energy densities and subsequently exciton densities reported in this paper.

Angle-resolved emission from the cavities is measured following the method of Richard et al. [18]. Emission from the cavity is collected with a $20 \times(0.45$ numerical aperture $)$ objective, and the Fourier plane of the objective is imaged onto the slit of a CCD spectrograph to obtain a two-dimensional dispersion (energy vs angle).

Time-resolved measurements of cavity emission are performed using a Kerr shutter-gating technique following the method of Kinoshita et al. [19]. The output of the optical parametric amplifier $(\lambda=532 \mathrm{~nm}, 1-\mathrm{kHz}$ repetition rate) is sent through an optical delay line and then is focused onto the surface of the sample with a $50 \times$ objective lens to a spot diameter of $20 \mu \mathrm{m}$. The photoluminescence (PL) emission is collected with the same objective lens, passes through a dichroic mirror, and is imaged onto a $\mathrm{SrTiO}_{3}$ crystal. In the absence of the gate beam, the PL is cross polarized between two polarizers, one before and one after the $\mathrm{SrTiO}_{3}$ crystal, with an attenuation of $\sim 10^{-6}$. The PL image spot is spatially overlapped in the crystal with a gate beam of wavelength $\lambda=$ $800 \mathrm{~nm}$, polarized at $45^{\circ}$ relative to the PL. In the presence of the gate beam, the polarization of the PL signal in that time slice is rotated, passes through the crossed polarizer, and then is imaged onto the slit of a CCD spectrograph. The delay of the excitation beam is scanned to obtain a time-resolved and spectrally resolved PL trace. The fall time of the Kerr medium response is found to be $250 \mathrm{fs}$ by performing a time-resolved measurement of the $80-$ fs excitation pulses directly.

\section{RESULTS AND DISCUSSION}

\section{A. Lasing threshold reduction}

The low- $Q$ microcavity $(Q=300)$ of $\lambda$ thickness is tested in regimes of slow excitation with $\tau_{\text {pump }} \sim 2 \tau_{\text {sp }}$ and fast excitation with $\tau_{\text {pump }} \sim T_{2} \ll \tau_{\mathrm{sp}}$, where $T_{2}=50-200 \mathrm{fs}$ is the optical dephasing time of typical organic dyes at room temperature $[13,20,21]$ and $\tau_{\mathrm{sp}}=3 \mathrm{~ns}$ is the spontaneous emission lifetime of DCM molecules in the $\mathrm{Alq}_{3}$ thin film. Under slow excitation ( $\tau_{\text {pump }}=8 \mathrm{~ns}$ ), the absorbed energy density at the lasing threshold is $80 \pm 4 \mu \mathrm{J} / \mathrm{cm}^{2}$ [Fig. 1(b)]. From Eq. (1) the expected threshold exciton density is $n_{\text {th }}=$ $3.0 \times 10^{18} \mathrm{~cm}^{-3}$ corresponding to an absorbed energy density of $39 \mu \mathrm{J} / \mathrm{cm}^{2}$. This predicted energy density agrees well with the measured threshold when we consider that the slow excitation duration $\tau_{\mathrm{pump}}$ is $\sim 2$ times longer than $\tau_{\mathrm{sp}}$, which reduces the energy absorbed per spontaneous emission lifetime. When the same cavity is tested under fast excitation $\left(\tau_{\text {pump }}=80 \mathrm{fs}\right)$, it exhibits absorbed energy lasing thresholds of $4 \pm 0.2 \mu \mathrm{J} / \mathrm{cm}^{2}\left(n_{\text {th }}=3 \times 10^{17} \mathrm{~cm}^{-3}\right)$, an over 20 -fold reduction in lasing threshold as compared to slow excitation. The observed threshold under fast excitation is ten times below what is expected from the conventional lasing threshold (a)

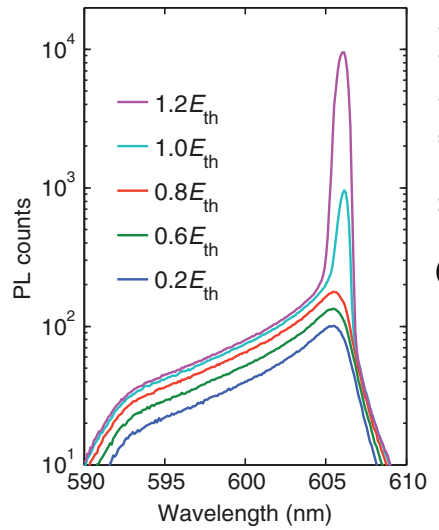

(b)
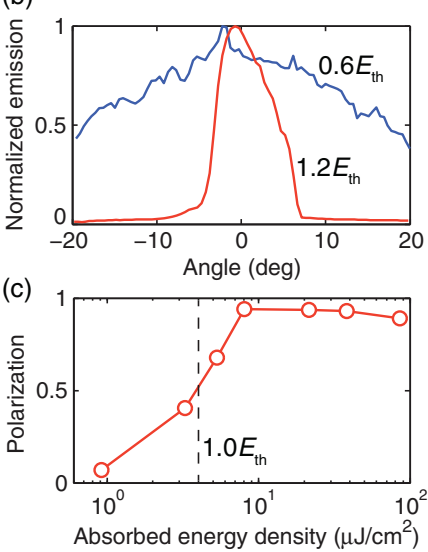

FIG. 2. (Color online) Cavity emission under fast (80 fs) excitation for the $Q=300$ cavity. (a) Emission spectra collected over angles $-20^{\circ}$ to $20^{\circ}$ at increasing excitation densities, showing a spectral line narrowing to $1 \mathrm{~nm}$ above threshold where $E_{\mathrm{th}}$ is the threshold energy density. (b) A wide emission cone below threshold narrows to a width of $8^{\circ}$ above threshold. (c) The degree of polarization of the emission $\left(T_{M}-T_{E}\right) /\left(T_{M}-T_{E}\right)$ as a function of excitation density shows highly polarized (0.94) emission above threshold.

condition [Eq. (1)], which predicts that the threshold should be independent of the excitation rate as long as $\tau_{\text {pump }}<\tau_{\mathrm{sp}}$.

A similar reduction in threshold is observed for a high- $Q$ microcavity $(Q=3000)$. Under slow excitation the observed energy threshold is $4.0 \pm 0.5 \mu \mathrm{J} / \mathrm{cm}^{2}$, whereas under fast excitation the threshold is $0.4 \pm 0.5 \mu \mathrm{J} / \mathrm{cm}^{2}$. As with the low$Q$ cavity, the predicted threshold from Eq. (1) $\left(3.9 \mu \mathrm{J} / \mathrm{cm}^{2}\right)$ is in good agreement with the threshold observed under slow excitation but not under fast excitation.

In addition to the threshold behavior, the emission of the cavities under fast excitation exhibits characteristic features of lasing (Fig. 2). For the low- $Q$ cavity, spectrally resolved emission is peaked at the resonance of $\lambda=605 \mathrm{~nm}$ with a linewidth of $2.5 \mathrm{~nm}$ below threshold and a long 15-nm blue tail corresponding to off-angle emission [Fig. 2(a)]. A similar linewidth above threshold is observed under slow excitation. Above threshold, the spectral linewidth narrows to $1 \mathrm{~nm}$, and the angular width of the emission peak narrows from $\pm 30^{\circ}$ to $\pm 4^{\circ}$ [Fig. 2(b)]. Furthermore, at threshold the emission becomes highly polarized in the $T_{M}$ direction along the pump polarization, a signature of lasing from amorphous organic materials in solid-state microcavity lasers [22] [Fig. 2(c)]. This behavior indicates that, although the cavity is far below the threshold density for conventional lasing, the observed transition exhibits the spectral, angular, and polarization features characteristic of coherence. Similar behavior is observed for the high- $Q$ cavity.

Several possible explanations for threshold reduction are first ruled out. The reduction is likely not due to fast excitation outcompeting the intersystem crossing to triplet states since lasing can be sustained for up to $50 \mathrm{~ns}$ at similar excitation densities [23] without a scheme for removal of triplet excitons [24]. The reduction in threshold also cannot be attributed to small microcavity effects [25] because the reduction is observed to be independent of cavity length. The same low- $Q$ 


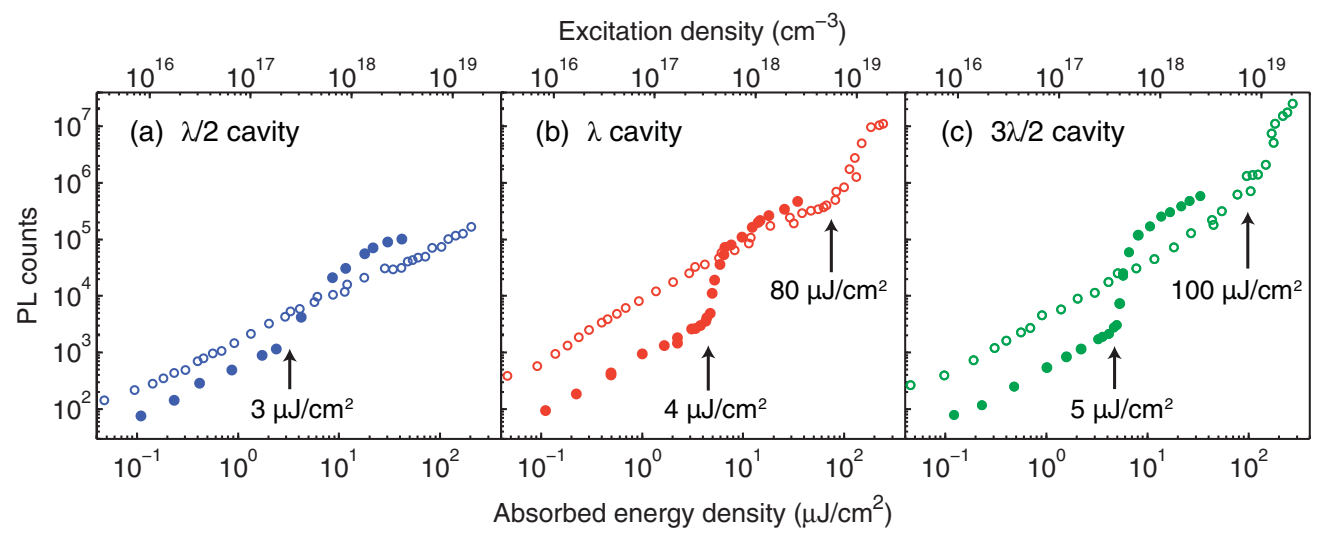

FIG. 3. (Color online) Threshold reduction under fast excitation for three cavity lengths with $Q \sim 300$. (a) $\lambda / 2$ cavity, (b) $\lambda$ cavity, and (c) $3 \lambda / 2$ cavity. Open circles are data for slow (8-ns) excitation, and filled circles are for fast (80-fs) excitation.

microcavity as described above is fabricated with lengths of $\lambda / 2, \lambda$, and $3 \lambda / 2$. Figure 3 shows that for the $\lambda$ and $3 \lambda / 2$ cavities, we observe a similar 20 -fold reduction in lasing threshold under fast excitation. The $\lambda / 2$-thickness cavity does not exhibit a lasing threshold under slow pumping because the required threshold exciton density of $6 \times 10^{18} \mathrm{~cm}^{-3}$ would be close to the DCM molecule density $n_{\mathrm{DCM}}=2.5 \times$ $10^{19} \mathrm{~cm}^{-3}$, thus requiring almost complete saturation and because a larger fraction of DCM excitons are quenched by the metal mirror compared to longer cavities. One possibility that we could not completely rule out is that the reduced thresholds are due to pump-induced stimulated Raman coherence. However, this mechanism is unlikely because the cavities are excited at $\lambda=532 \mathrm{~nm}$, which is far away energetically from the microcavity emission resonance at $\lambda \approx 605 \mathrm{~nm}$.

As a possible mechanism, we consider the unexpectedly low lasing threshold density in the context of super-radiant cooperative emission. As known from the Dicke SR model [9], a collective system of excitons radiates at an enhanced rate given by [12]

$$
\frac{1}{\tau_{R}}=\frac{1}{\tau_{\mathrm{sp}}} \frac{n \lambda^{2} L}{8 \pi},
$$

where $n$ is the volume density of excitons, $L$ is the length of the sample, and $\lambda=\lambda_{0} / \eta$ is the emission wavelength $\lambda_{0}$ reduced by the refractive index $\eta$ of the material. It has been shown that in an optical cavity the effective length of the material is increased, which enhances the SR rate in proportion to the finesse of the cavity [11]. Björk et al. [14] have calculated the overlap between the SR emission and a microcavity mode to show that the enhancement factor is given by $\left(1+\sqrt{R_{\text {eff }}}\right) /\left(1-\sqrt{R_{\text {eff }}}\right)$, where $R_{\text {eff }}$ is the geometric average of the microcavity mirror reflectivities. For the low- $Q$ cavity, $R_{\text {eff }}=0.98$, and the enhancement factor is 190. Although SR effects could be observed in thin-film samples, the presence of the cavity substantially reduces the required exciton density. Furthermore, the SR rate is modified by the mismatch between the cavity spectral linewidth and the inhomogeneous linewidth of DCM emission. This mismatch results in only a fraction of the excitons $T_{2}^{*} / \tau_{c}$ being coupled to the cavity mode, where $T_{2}^{*}=2.8 \mathrm{fs}$ is the inhomogeneous dephasing time of DCM obtained from the emission spectral width and $\tau_{c}=95 \mathrm{fs}$ is the photon lifetime of the low- $Q$ cavity. Finally, the expression for the cavity-enhanced SR rate in the $\lambda$-thickness cavity is

$$
\frac{1}{\tau_{R}}=\frac{1}{\tau_{\mathrm{sp}}} \frac{n \lambda^{2} L}{8 \pi}\left(\frac{1+\sqrt{R_{\mathrm{eff}}}}{1-\sqrt{R_{\mathrm{eff}}}}\right) \frac{T_{2}^{*}}{\tau_{c}} .
$$

Therefore, at the threshold exciton density with fast excitation of $n_{\mathrm{th}}=3 \times 10^{17} \mathrm{~cm}^{-3}$, the SR time for the low- $Q$ cavity is $\tau_{R}=620 \pm 50$ fs. Likewise for the high- $Q$ cavity, the SR time is calculated to be $\tau_{R}=6200 \pm 500 \mathrm{fs}$. Using Eq. (3) for the SR rate and the expression for the stimulated emission cross section $\sigma_{\mathrm{SE}}=\lambda^{2} T_{2}^{*} / 8 \pi \tau_{\mathrm{sp}}$, the lasing threshold from Eq. (1) in the presence of SR is reduced according to $1 / \tau_{c}=n_{\mathrm{th}} c\left(\tau_{R} / \tau_{c}\right) \sigma_{\mathrm{SE}}$, where $\left(\tau_{R} / \tau_{c}\right) \sigma_{\mathrm{SE}}=7 \sigma_{\mathrm{SE}}$ is the enhanced stimulated emission cross section under conditions of SR.

The condition for "strong SR" in which all light is emitted coherently requires that the optical dephasing time is longer than the SR time $T_{2}>\tau_{R}$, which our system does not satisfy. However SR can still occur in the "limited SR" regime as discussed by MacGillivray and Feld [12] in which $T_{2}<\tau_{R}$. In such a situation only a fraction of emission occurs coherently. Typical $T_{2}$ values for organic laser dyes at room temperature have been measured to be in the range of 50-200 fs [13,20,21]. This value of $T_{2}$ and the SR time of $\tau_{R}=620 \pm 50 \mathrm{fs}$ sets the system in the limited SR regime under fast excitation at the observed threshold density of $n_{\text {th }}=3 \times 10^{17} \mathrm{~cm}^{-3}$ for the low- $Q$ cavity. For the high- $Q$ cavity, the SR time is substantially longer than the dephasing time, but as shown by Temnov and Woggon [26], SR can still occur when $T_{2}<\tau_{R}$. Indeed, in both cavities only a fraction of emitters have enough phase coherence to lock their spontaneous emission. However, once the SR emission from this subpopulation of molecules is injected into the cavity, it provides the necessary optical field to stimulate emission of the remainder of the excited molecules. This effect of super-radiance seeding the stimulated emission occurs because of the presence of the cavity. As a result of super-radiance and stimulated emission occurring in concert, a single threshold would be expected as observed.

The phase coherence imparted by the pump is likely rapidly lost due to the Franck-Condon shift and vibrational relaxation at early times after excitation. Hence, as in traditional superradiance work, the macroscopic dipole evolves spontaneously 
because the density of excitons is high enough relative to the dephasing time. Although the phase locks spontaneously, the orientation of the excited dipoles is determined by the pump laser. In other words, the excitation pulse polarization should select a subpopulation of DCM molecules that are excited most efficiently. We find that the output polarization above threshold follows the pump polarization as the linear pump polarization is rotated. This is not surprising given that the laser selects a subset of emitters with the same orientation of the transition dipole moment as the pump polarization. However, although the dipoles can have the same orientation, phase coherence (or lack of it) is determined by the length of the pump pulse, not its polarization.

\section{B. Conventional laser theory}

To contrast the observed threshold reduction with conventional lasing theory, the DCM microcavities are modeled using the rate equation formalism described by Koschorreck et al. [5]. The dynamics of the DCM microcavities are modeled by considering the four-level structure of DCM, which in our experiments is optically pumped directly, without a Förster resonant energy transfer from the $\mathrm{Alq}_{3}$ host matrix. The energy-level diagram of DCM is shown in Fig. 4 in which $m$ is the population of the pump state, $n$ is the population of the relaxed excited state, and $q$ is the number of photons in the cavity mode. To model the dynamics of this system, we consider the coupled differential equations describing the three species following the notation of Brückner et al. [2],

$$
\begin{aligned}
\frac{d m}{d t} & =p\left(m_{\mathrm{tot}}-2 m\right)-k_{\mathrm{vib}} m, \\
\frac{d n}{d t} & =k_{\mathrm{vib}} m-(1-\beta) A_{f} n-\beta A_{c}(1+q) n-\Gamma n, \\
\frac{d q}{d t} & =\beta A_{c}(1+q) n-\gamma q,
\end{aligned}
$$

where $p$ is the normalized pump rate, $m_{\text {tot }}$ is the total number of DCM molecules in the volume of one cavity mode, $k_{\mathrm{vib}}$ is the vibrational relaxation rate, $\beta$ is the spontaneous emission coupling factor into the cavity, $A_{f}$ is the radiative rate into free space, $A_{f}$ is the cavity-enhanced radiative rate into the cavity mode, $\Gamma$ is the nonradiative decay rate of the relaxed excited state $n$, and $\gamma$ is the leakage rate of photons from the cavity. The populations, $m, n$, and $p$, are per mode volume.

The equation for the pump state population $m$ includes a saturation term determined by the total number of molecules

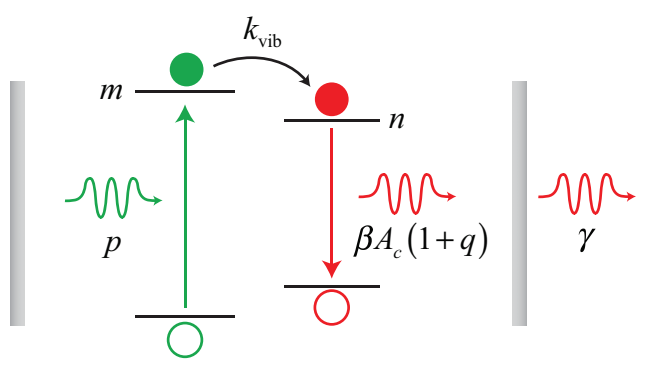

FIG. 4. (Color online) Energy-level diagram of DCM. The pump state is $m$, and the fluorescent state is $n$. within the volume of the cavity mode $V$. The mode volume is $V=r_{\text {eff }}^{2} L$, where $L=350 \mathrm{~nm}$ is the thickness of the cavity gain layer for a $\lambda$-thickness cavity and $r_{\text {eff }}$ is the effective transverse-mode radius. The effective mode radius is given by [27]

$$
r_{\mathrm{eff}}=\left[\frac{\pi L \lambda_{0} \sqrt{R_{\mathrm{eff}}}}{8 n\left(1-R_{\mathrm{eff}}\right)}\right]^{1 / 2},
$$

where $\lambda_{0}=600 \mathrm{~nm}$ is the cavity resonance free space wavelength, $n=1.62$ is the cavity refractive index, $L=350 \mathrm{~nm}$ is the cavity length, and $R_{\text {eff }}=0.98$ is the geometric mean of the cavity mirror reflectivities for the low- $Q$ cavity. From these values we find $r_{\text {eff }}=1.5 \mu \mathrm{m}$. Based on the $2.5 \%$ doping of DCM in $\mathrm{Alq}_{3}$ and the molecular weights of each molecule, the estimated two-dimensional density of DCM molecules is $N_{D}=1 \times 10^{15} \mathrm{~cm}^{-2}$. Consequently, the number of molecules per mode volume is $m_{\text {tot }}=\pi r_{\text {eff }}^{2} N_{D}=7 \times 10^{7}$.

The vibrational relaxation rate from the pump state to the relaxed excited state is assumed to be rapid with $k_{\mathrm{vib}}=$ $1 /(500 \mathrm{fs})[2,22]$, although changes in this parameter did not affect the results significantly. The spontaneous emission coupling factor is estimated to be $\beta=0.002$ following the results of Björk et al. [28] using the linewidths of the cavity $\left(\Delta \lambda_{\text {cav }}=2 \mathrm{~nm}\right)$ and DCM emission $\left(\Delta \lambda_{D}=80 \mathrm{~nm}\right)$. For the nonradiative decay rate we use $\Gamma=1 /(6 \mathrm{~ns})$, and the radiative rate into free space is $A_{f}=1 /(3 \mathrm{~ns})$. The cavity-enhanced radiative rate is $A_{c}=F_{P} A_{f}$, where $F_{P}$ is the Purcell factor given by [29]

$$
F_{P}=\frac{3 Q\left(\lambda_{0} / n\right)^{3}}{4 \pi^{2} V} .
$$

Using the parameters for the $Q=300$ cavity, we find $F_{P}=1.9$ and consequently $A_{c}=1 /(1.6 \mathrm{~ns})$. The cavity photon escape rate $\gamma$ is determined from the cavity lifetime, which is given by $\tau_{c}=Q \lambda_{0} / 2 \pi c$, where $c$ is the speed of light. For the cavity decay rate, $\gamma=1 /(95 \mathrm{fs})$.

The dynamics of the cavity emission are simulated by numerically solving Eqs. (4). The initial conditions for the simulation are that the DCM molecules are in the ground state with no intracavity photons, $m=0, n=0$, and $q=0$. The excitation is a time-dependent pump term,

$$
p(t)=p_{0} \exp \left(-\frac{t^{2}}{2 \sigma_{p}^{2}}\right) .
$$

The full width of the pulse at $1 / e$ intensity is $\tau_{\text {pump }}=2 \sqrt{2} \sigma_{p}$, and this is the value referred to as the pulse width. The total energy delivered by one pulse per unit area is then calculated from

$$
E_{p}=\frac{h c / \lambda_{p}}{\pi r_{\mathrm{eff}}^{2}} \int_{-\infty}^{\infty} p(t)\left(m_{\mathrm{tot}}-2 m\right) d t,
$$

where $h c / \lambda_{p}$ is the energy of one pump photon.

The simulated dynamics of the low- $Q$ cavity emission are shown below and above the nonlinear threshold in the two excitation regimes $\tau_{\text {pump }}=100 \mathrm{fs}$ (fast) and $\tau_{\text {pump }}=1 \mathrm{~ns}$ (slow) (Fig. 5). Under both kinds of excitation, the lifetime of the emission pulse is determined by the pump pulse width. Under even shorter excitation, the emission pulse width becomes limited by the cavity photon lifetime. 
(a)

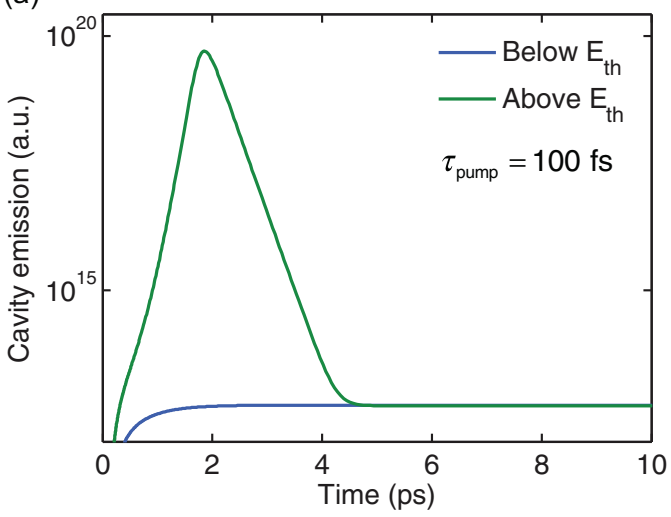

(b)

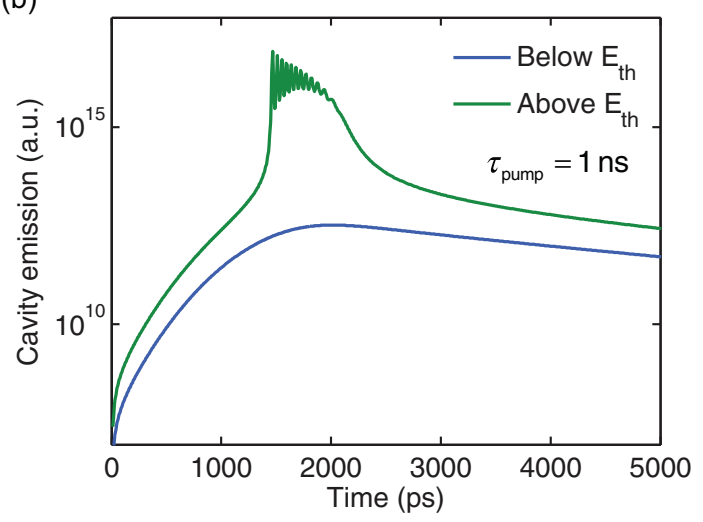

FIG. 5. (Color online) Comparison of cavity dynamics with (a) 100-fs excitation pulse width, and (b) 1-ns excitation pulse width. The emission pulse is offset from $t=0$ because the excitation pulse has a positive offset to avoid negative time values in the simulation. The oscillations above threshold are due to the repumping of state $n$ from state $m$ due to vibrational relaxation.

Figure 6 shows the simulated time-integrated cavity emission as a function of absorbed energy density with different excitation pulse widths. The regimes of spontaneous emission, lasing, and saturation are seen for all the simulated pump pulse widths. The lasing threshold is defined by the inflection point of the input-output dependence as shown with the dashed line in Fig. 6. Despite the widely varying duration of the excitation and subsequent emission pulses, the input-output curves are mostly insensitive to changes in pump pulse width. Figure 7 shows how the predicted lasing threshold depends on $\tau_{\text {pump. }}$. The modest increase in the simulated threshold density for $\tau_{\text {pump }}>100$ ps occurs because the excitation pulse length approaches the radiative lifetime of the excitons $\tau_{\mathrm{sp}}$.

\section{Excitation pulse-width dependence}

To compare the behavior of the DCM microcavity to conventional laser theory and to SR theory, we explored the onset of threshold reduction by experimentally varying the excitation time $\tau_{\text {pump }}$. Figure 7 shows that for the low- $Q$ cavity, the threshold energy density for lasing decreases dramatically from $n_{\text {th }}=4 \times 10^{18} \mathrm{~cm}^{-3}$ when $\tau_{\text {pump }}=900$ ps to $n_{\text {th }}=$ $3 \times 10^{17} \mathrm{~cm}^{-3}$ when $\tau_{\text {pump }}=1$ ps. This strong dependence on

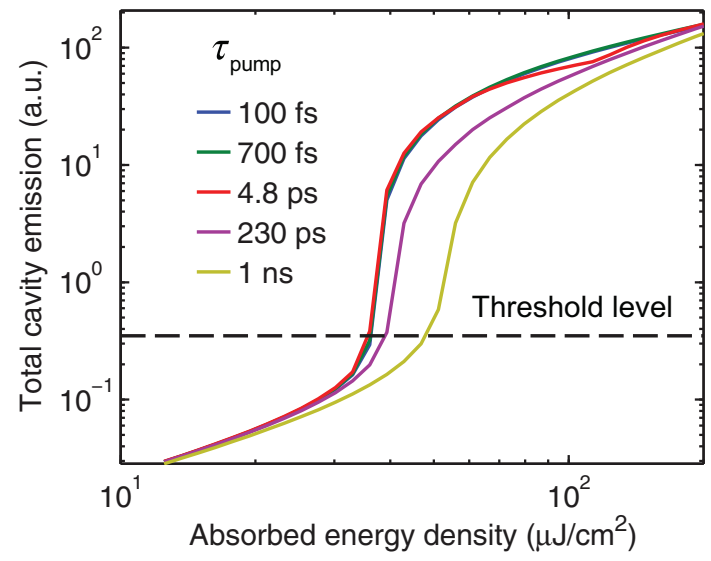

FIG. 6. (Color online) Simulated cavity emission as a function of pump energy density for a range of pump pulse widths. The dashed line indicates the level at which the threshold is determined. excitation pulse width is in stark contrast to the predictions of conventional laser theory. The observed threshold is constant as the excitation pulse width is reduced to below 1 ps because the excitations are created in a time shorter than the SR time $\tau_{\text {pump }} \leqslant \tau_{R}$, suggesting that SR may be a valid interpretation. With 80 -fs pulsed excitation, the ratio of the simulated to the observed threshold densities is 7 , which agrees well with the enhancement of the effective stimulated emission cross section $\left(\tau_{R} / \tau_{c}\right) \sigma_{\mathrm{SE}} \approx 7 \sigma_{\mathrm{SE}}$.

\section{Emission dynamics}

Finally, the temporal dynamics of the low- $Q$ microcavity emission are observed using a Kerr gating technique with 250 -fs resolution. At a density of $2 n_{\text {th }}=6 \times 10^{17} \mathrm{~cm}^{-3}$ [Fig. 8(d)], the emitted pulse is peaked at 4 ps after excitation and has a decay time constant of $1100 \mathrm{fs}$, which is $\sim 2 \tau_{R}$, as expected from the typical hyperbolic secant shape of SR pulses [14]. As the excitation density is increased to $3 n_{\text {th }}$ [Fig. 8(f)], the decay time constant is reduced to $690 \mathrm{fs}$, proportional with

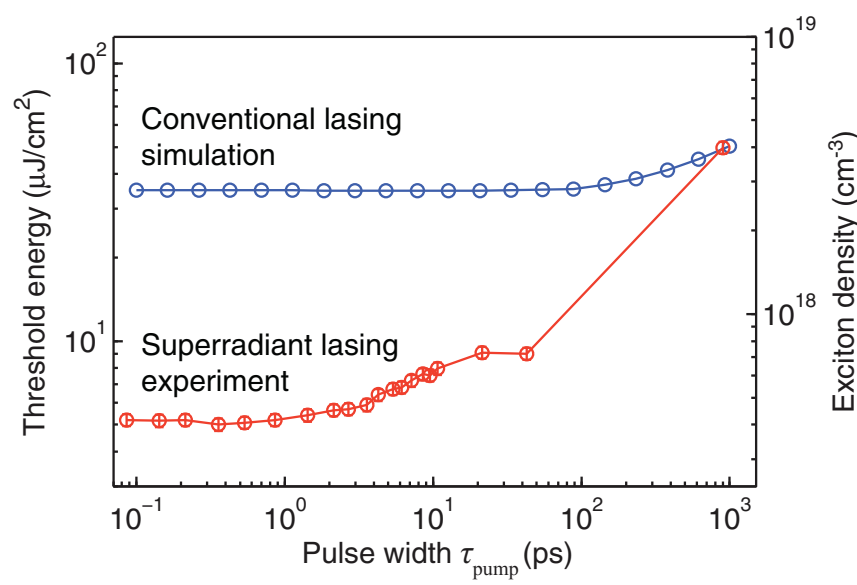

FIG. 7. (Color online) Threshold energy density as a function of excitation pulse width for the low- $Q$ cavity. Experiment shows a dramatic reduction in threshold energy density for shorter pulses, whereas simulation of the conventional lasing threshold does not show the same dependence, indicating that phenomena beyond conventional lasing are responsible for the reduced threshold. 
(a)
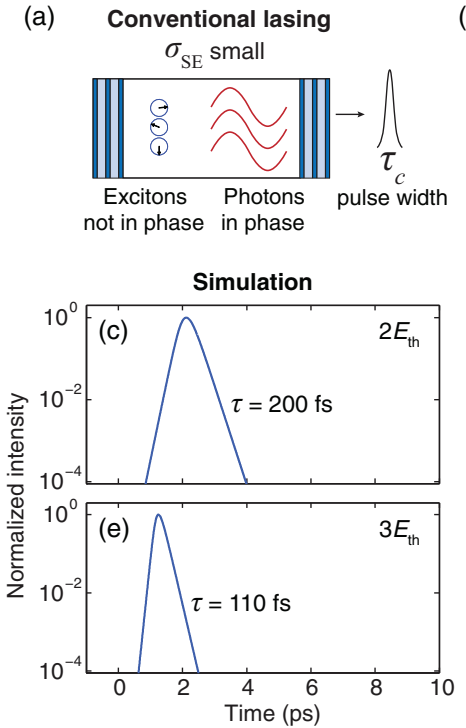

(b) Super-radiant lasing
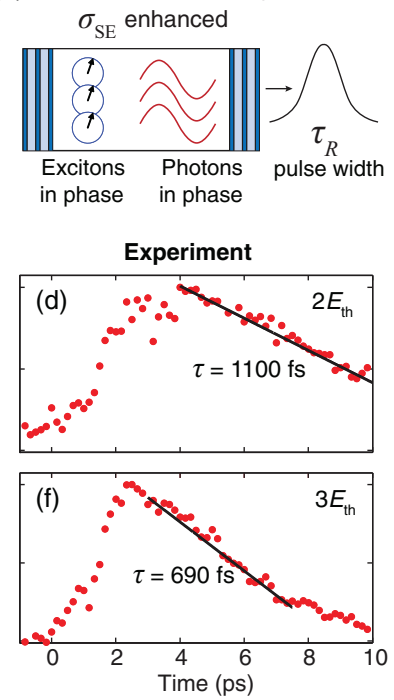

FIG. 8. (Color online) (a) For regular lasing ( $\tau_{\text {pump }}=8 \mathrm{~ns}$ ), photons in the microcavity are in phase, whereas the radiating excitons are not, and under pulsed excitation, the emission pulse is determined by the cavity lifetime $\tau_{c}$. (b) In super-radiant lasing ( $\tau_{\text {pump }}=80 \mathrm{fs}$ ), the excitons are also coherently coupled to one another and hence radiate with an effective cross section enhanced by $\tau_{R} / \tau_{c}$, and the emission pulse is determined by the SR time $\tau_{R}$, resulting in a reduced lasing threshold excitation density. (c)-(f) Simulated and measured emissions from the low- $Q$ cavity following 80 -fs pulsed excitation. The pulse decay times predicted for the conventional laser model are (c) $200 \mathrm{fs}$ for excitation density $2 E_{\mathrm{th}}$ and (e) $110 \mathrm{fs}$ for excitation density $3 E_{\mathrm{th}}$, approaching $\tau_{c}=95$ fs for high excitation densities. In contrast, the measured pulse decay times above threshold are as follows: (d) $1100 \mathrm{fs}$ for excitation density $2 E_{\text {th }}$ and (f) $690 \mathrm{fs}$ for excitation density $3 E_{\mathrm{th}}$. The observed pulse decay times are $\sim 2 \tau_{R}$, in agreement with the SR regime of operation.

the decrease in $\tau_{R}$. By comparison, the microcavity emission as modeled by rate equations of the conventional laser under the same excitation conditions [Figs. 8(c) and 8(e)] shows decay time constants of 200 and 110 fs for the two excitation densities, respectively. These decay times approach the cavity photon lifetime $\tau_{c}$ with increasing density, in contrast to the measured pulses which follow the SR time. Likewise, the measured time-resolved decay from the high- $Q$ microcavity shows a decay time constant of 5900 fs (Fig. 9), in good agreement with the SR time of $\tau_{R}=6200 \pm 500 \mathrm{fs}$ predicted by Eq. (3). This decay time is much longer than the cavity photon lifetime of $950 \mathrm{fs}$, which would determine the cavity decay time under conventional lasing.

\section{CONCLUSIONS}

In conclusion, we observed an unexpected tenfold reduction in lasing threshold of an organic vertical microcavity under subpicosecond excitation. The threshold reduction is

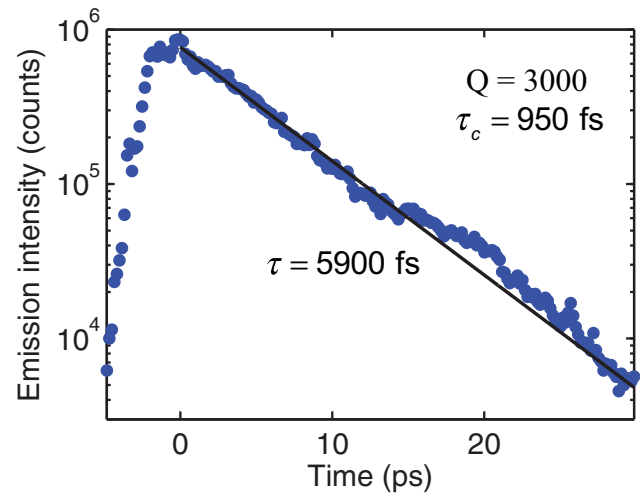

FIG. 9. (Color online) Time-resolved emission from the high- $Q$ cavity above threshold under 80 -fs excitation showing a decay time constant of $5900 \mathrm{fs}$, in good agreement with the SR time predicted by Eq. (3).

interpreted in the context of super-radiant lasing based on the predicted SR time and the homogeneous dephasing time of DCM. SR is further supported by the observed temporal dynamics of the emission, which agrees with SR pulse dynamics, and differs substantially from the predictions of a conventional lasing model. To show the generality of the phenomenon, the threshold reduction is demonstrated in both low- $Q$ (300) and high- $Q$ (3000) microcavities. The proposed SR lasing mechanism is a consequence of the rapid relaxation of the four-level organic lasing material and could be observed more generally in other materials and geometries, such as microring resonators and distributed feedback lasers $[1,30]$. The consistent agreement in this study between the experiments and the SR theory suggests that cooperative exciton emission can be an important effect even at room temperature. As a result, studies of organic lasing structures, both in the weak-coupling and in the strong-coupling regimes, should take into consideration the possible contribution of super-radiant effects. Furthermore, SR can be harnessed to reduce the threshold of lasing chemosensors [31,32], thus decreasing the amount of gain material needed for lasing and increasing sensitivity.

\section{ACKNOWLEDGMENTS}

The authors gratefully acknowledge insightful conversations with Professor M. Baldo and support from the Center for Excitonics, an Energy Frontier Research Center funded by the U.S. Department of Energy under Grant No. DE-SC0001088. Measurements were performed in the MIT Nanostructured Materials Metrology Laboratory within the NSF MRSEC Center for Materials Science and Engineering on equipment provided by the Eni-MIT Solar Frontiers Center. The authors also acknowledge Dr. S. Kooi of the MIT Institute for Soldier Nanotechnologies for performing the cross-sectional TEM of the microcavity laser sample. G.M.A. acknowledges support from the Hertz Foundation and an NSF Graduate Research Fellowship.
[1] I. D. W. Samuel and G. A. Turnbull, Chem. Rev. 107, 1272 (2007).
[2] R. Brückner, M. Sudzius, H. Fröb, V. G. Lyssenko, and K. Leo, J. Appl. Phys. 109, 103116 (2011). 
[3] V. G. Kozlov, V. Bulović, P. E. Burrows, and S. R. Forrest, Nature (London) 389, 362 (1997).

[4] M. D. McGehee, M. A. Díaz-García, F. Hide, R. Gupta, E. K. Miller, D. Moses, and A. J. Heeger, Appl. Phys. Lett. 72, 1536 (1998).

[5] M. Koschorreck, R. Gehlhaar, V. G. Lyssenko, M. Swoboda, M. Hoffmann, and K. Leo, Appl. Phys. Lett. 87, 181108 (2005).

[6] H. Deng, H. Haug, and Y. Yamamoto, Rev. Mod. Phys. 82, 1489 (2010).

[7] S. Kéna-Cohen and S. R. Forrest, Nat. Photonics 4, 371 (2010).

[8] J. D. Plumhof, T. Stöferle, L. Mai, U. Scherf, and R. F. Mahrt, Nature Mater. 13, 247 (2014).

[9] R. H. Dicke, Phys. Rev. 93, 99 (1954).

[10] B. E. A. Saleh and M. C. Teich, Fundamentals of Photonics, 2nd ed. (Wiley-Interscience, Hoboken, NJ, 2007).

[11] L. Moi, P. Goy, M. Gross, J. M. Raimond, C. Fabre, and S. Haroche, Phys. Rev. A 27, 2043 (1983).

[12] J. C. MacGillivray and M. S. Feld, Phys. Rev. A 14, 1169 (1976).

[13] S. V. Frolov, W. Gellermann, M. Ozaki, K. Yoshino, and Z. V. Vardeny, Phys. Rev. Lett. 78, 729 (1997).

[14] G. Björk, S. Pau, J. Jacobson, and Y. Yamamoto, Phys. Rev. B 50, 17336 (1994).

[15] M. S. Bradley, J. R. Tischler, Y. Shirasaki, and V. Bulović, Phys. Rev. B 78, 193305 (2008).

[16] A. M. Weiner, J. P. Heritage, and E. M. Kirschner, J. Opt. Soc. Am. B 5, 1563 (1988).

[17] P. van der Meulen, H. Zhang, A. M. Jonkman, and M. Glasbeek, J. Phys. Chem. 100, 5367 (1996).
[18] M. Richard, J. Kasprzak, R. Romestain, R. André, and L. S. Dang, Phys. Rev. Lett. 94, 187401 (2005).

[19] S. Kinoshita, H. Ozawa, Y. Kanematsu, I. Tanaka, N. Sugimoto, and S. Fujiwara, Rev. Sci. Instrum. 71, 3317 (2000).

[20] M. D. Barnes, W. B. Whitten, S. Arnold, and J. M. Ramsey, J. Chem. Phys. 97, 7842 (1992).

[21] C. H. Brito Cruz, R. L. Fork, W. H. Knox, and C. V. Shank, Chem. Phys. Lett. 132, 341 (1986).

[22] V. G. Kozlov, V. Bulovic, P. E. Burrows, M. Baldo, V. B. Khalfin, G. Parthasarthy, S. R. Forrest, Y. You, and M. E. Thompson, J. Appl. Phys. 84, 4096 (1998).

[23] N. C. Giebink and S. R. Forrest, Phys. Rev. B 79, 073302 (2009).

[24] Y. Zhang and S. R. Forrest, Phys. Rev. B 84, 241301(R) (2011).

[25] F. De Martini and G. R. Jacobovitz, Phys. Rev. Lett. 60, 1711 (1988).

[26] V. V. Temnov and U. Woggon, Phys. Rev. Lett. 95, 243602 (2005).

[27] T. Enomoto, T. Sasaki, K. Sekiguchi, Y. Okada, and K. Ujihara, J. Appl. Phys. 80, 6595 (1996).

[28] G. Björk, H. Heitmann, and Y. Yamamoto, Phys. Rev. A 47, 4451 (1993).

[29] A. Kavokin, J. J. Baumberg, G. Malpuech, and F. P. Laussy, Microcavities (Oxford University Press, Oxford, 2011).

[30] S. Chénais and S. Forget, Polym. Int. 61, 390 (2012).

[31] S. W. Thomas III, G. D. Joly, and T. M. Swager, Chem. Rev. 107, 1339 (2007).

[32] A. Rose, Z. Zhu, C. F. Madigan, T. M. Swager, and V. Bulović, Nature (London) 434, 876 (2005). 